\title{
A Study of Bacterial Isolates in Diarrhea Infection in Pediatric Patients Under 5 Yrs. of Age Group And Their Antibiotic Susceptibility Pattern, Admitted At P.D.U. Govt. Hospital, Rajkot, Gujarat, India.
}

\author{
Dr. ShwetaChouhan ${ }^{1}$ Dr. Anil Chaudhary ${ }^{2}$ Dr. Saurabh Pandey ${ }^{3,}$ \\ Dr. Madhulika Mistry ${ }^{4}$ Dr. YogeshGoswami ${ }^{5}$ \\ ${ }^{1}$ Consultant Microbiologist, SRL Diagnostics, Mumbai, India. \\ ${ }^{2}$ Microbiologist at General Hospital, Deesa, Gujarat, India. \\ ${ }^{3}$ Clinical Associates, Jaslok Hospital, Mumbai, India. \\ ${ }^{4}$ Associate professor, P.D.U Medical college, Rajkot, Gujarat, India. \\ ${ }^{5}$ Dean, P.D.U Medical college, Rajkot, Gujarat, India.
}

\begin{abstract}
Background: Diarrhea is a major cause of mortality \& morbidity in pediatric age group of patients' in developing countries. Surveillance for a broad range of enteric pathogens is necessary to accurately predict the frequency of pathogens \& potential changes in antibiotic resistance pattern.

Methods: The Stool samples were collected between January 2014 to January 2015 from a total of 200 outdoor \& indoor patients at P.D.U. Medical College \& Govt. hospital. Bacterial pathogens were isolated \& antibiotic susceptibility patterns were ascertained. For E.coli isolates genotyping was done at NICED, Kolkata.

Result: The bacterial isolates were $54.5 \%$. Isolation rates were higher in male compared to females, \& in 2 years of age group. The bacterial isolation rates were EAEC 80\%, ETEC 10\%, EPEC 10\%, S.sonnei 0.5\%.Maxium resistance were observed to Cephalothin, Ampicillin, Cefotaxime, Cephazolin.

Conclusion: The bacterial pathogens were found to be a significant cause of diarrhea. The study highlights the need for appropriate treatment of children with diarrhea and promotion of its prevention.

Keywords: Clinical Laboratory Standard Institute (CLSI),E.coli, Entero Aggregative E.coli(EAEC), Entero Pathogenic E.coli (EPEC),Entero Toxigenic E.coli (ETEC),Entero Invasive E.coli (EIEC).

\section{Introduction}

Diarrhea is a major public health problem in developing countries. An estimate of 1.5 billion episodes of diarrhea occur each year and 3 million children under the age of 5 years die due to diarrhea. ${ }^{[1]}$ Its effect is particularly devastating on children below 2 years of age, with eighty percent $(80 \%)$ of deaths due to diarrhea occurring during the first 2 years of life. ${ }^{[2]}$ Two recent studies presented global estimates of child deaths due to diarrhea that were equal to 2.5 million $^{[3]}$ and 2.1 million. $^{[4]} \mathrm{A}$ third review has estimated that $22 \%$ of all deaths among under-5s in sub -Saharan Africa and 23\% in south Asia were caused by diarrheal diseases in the year $2000 .{ }^{[5]}$ Diarrhea is a particularly deadly illness for young children, with acute diarrhea liable to cause death within a day or less if left untreated. ${ }^{[6]}$ Diarrheal diseases remain a serious threat to child survival; with an estimated 9\% of children suffering from this condition in India. ${ }^{[6]}$ Diarrheal diseases account for $15.4 \%$ of under 5 population in India which includes $16.5 \%$ of rural and $12.4 \%$ of urban population. ${ }^{[7]}$ In India diarrheal diseases account for 1.7 episodes per child per year in under 5 population. ${ }^{[8]}$
\end{abstract}

\section{Materials And Methods}

As a part of a passive-surveillance study conducted between January 2014 to January 2015 in PDU Govt. Medical College, Rajkot. A total number of 200 stool samples from children suffering from diarrhea, aged $\leq 60$ months were collected. Pediatric outer patient department and indoor patient of pediatric ward of the hospital were involved in this study. Children enrolled in the study were identified as diarrheal cases, characterized by the occurrence of three or more loose, liquid or watery stools, or at least one bloody, loose stool, in the preceding 3 day period.

Stools samples collected in sterile screw-capped plastic containers were transported to the Department of Microbiology at PDU govt. Medical College Rajkot, on the day of collection, where they were processed initially by standard culture and identification methods.Routine microscopy by saline /iodine preparation reveals presence of pus cells, fat globules, red blood cells and ova and/or cyst of parasite .Hanging drop preparations for visualizing the motility of bacteria.After this the samples were inoculated on several media for maximum yield. 
Including blood agar, MacConkey agar and Nutrient agar and selective media like salmonella -shigella agar, TCBS agar. Alkaline peptone water was used as the enrichment medium for vibrio species. The inoculated media were incubated at $37^{\circ} \mathrm{C}$ overnight. The colonies were examined by biochemical reactions. ( Indole Production, M.R., Citrate, Sugar fermentation, PPA Hydrolysis, Urease Production, Triple Sugar Iron Test) and agglutination with specific antisera. The antibiotic susceptibility testing of the pathogens isolated from the clinical specimen against different antibiotics were done using Kirby Bauer method of disc diffusion, the zone of inhibition was interpreted according to the CLSI guidelines. (CLSI 2015)

Genotyping: From each plate showing growth of E. coli, a full loop of E. coli-like colonies was stored at $-20^{\circ} \mathrm{C}$ in Nutrient Agar, until they were transported to the NICED KOLKATTA, for Genotype pattern typing. A one-step multiplex PCR assay using eight different specific primers was performed to determine the prevalence of five different DEC pathotypes in the E. coli flora. The criteria for determining the different types of DiarrheagenicE.coli (DEC) by PCR were as follows: the presence of eltB and/or estA markers for ETEC; the presence of $\mathrm{vt} 1$ and/or vt2 for EHEC (the presence also of eaeA confirmed the presence of a typical EHEC); the presence of bfpA and eaeA for typical EPEC, (only eaeA for atypical EPEC); the presence of ial for EIEC/Shigella; and the presence of pCVD432 for EAEC.A smear of each bacterial culture (the primary streak of E. coli-positive samples on the agar plates) was suspended in $1 \mathrm{ml}$ PBS (phosphate buffered saline) to a density of four McFarland standard $\left(1 \times 10^{9}-5 \times 10^{9}\right.$ bacteria/ml), boiled for 20 minutes, followed by centrifugation at 2,500 g for 10 minutes to pellet the cell debris. Two $\mu \mathrm{l}$ of DNA template was amplified in a final volume of $25 \mu \mathrm{l}$ reaction mixture using the pureTaq Ready-to-Go PCR Bead and containing $200 \mu \mathrm{M}$ dNTPs, $10 \mathrm{mMTris} / \mathrm{HCl}(\mathrm{pH}$ 9), $50 \mathrm{mMKCl}, 1.5 \mathrm{mM} \mathrm{MgCl} 2,2.5 \mathrm{U}$ pureTaq DNA polymerase, and $0.2 \mu \mathrm{M}$ of each primer mix .The PCR reactions were performed in a Gene Amp PCR system as follows: 1 cycle at $96^{\circ} \mathrm{C}$ for 4 min; 35 cycles at $94^{\circ} \mathrm{C}$ for $30 \mathrm{~s}, 58^{\circ} \mathrm{C}$ for $30 \mathrm{~s}$, and $72^{\circ} \mathrm{C}$ for $1 \mathrm{~min}$; ending with a 7 min extension at $72^{\circ} \mathrm{C}$. The amplified DNA products $(10 \mu \mathrm{l})$ were then electrophoresed on $1.5 \%$ agarose gel at $120 \mathrm{mV}$ for 40 minutes and visualized under UV light after staining with ethidium bromide. Products were sized against a $100 \mathrm{bp}$ DNA ladder used as a molecular weight marker. If the pooled DNA temple result was negative following gel electrophoresis, the sample was considered as negative for DEC. If the bands were seen after gel electrophoresis, the band sizes on the gel were compared with the size marker bands in order to identify the suspected DEC in the stool samples.

\section{Result}

During the study period from January 2014 to January 2015, total of 200 stool samples were examined from patient of pediatric age group; attended the Outpatient department and indoor patient of P.D.U. Govt. medical college \& hospital, Rajkot. Isolation rate of bacterial isolates in our study rate is 54.5\%. Out of 200 samples E.coli was present in 108 samples $(54 \%)$ while in one sample Shigellasonnei was isolated (0.5\%). [Table 1] Isolation of E.coli was higher in male than female in present study. Isolation of E.coli species was highest in up to 2 year age group.Klebsiella species are isolated which are considered as non- pathogenic.After doing genotyping out of 108 E.coli 10 were found diarrheogenic strains. Out of these 10, 8 were EAEC (80\%), 1 was ETEC (10\%) and 1 was EPEC (10\%). [Table 2]. E.coli resistance to Ampicillin was 94.4\%, Cefotaxime \& Cephalothin was $93.51 \%$, while least resistance to Meropenam $1.85 \%$, Piperacillin Tazobactum \& Ceftazidime Clavulanic acid was $19.44 \%$.[Table 3].

\section{Discussion}

In our study rate is $54.5 \%$, which was higher than sang et al $17.7 \%$, \&it was comparable with Ansari et al $50-60 \%$. Isolation rate from stool in this study was $0.5 \%$ which was nearly comparable to Sang et al ${ }^{[9]}$ $(2.3 \%)$. Isolation of E.coli was higher in male than female in present study, which is similar to Study done by Ansari et $\mathrm{al}^{[10]}$. Isolation of E.coli species was highest in up to 2 year age group, which is similar to study done by Ansari et al. In present study the EAEC was $80 \%$, which it is equal to study done by Sang et al (79.4\%). \& ETEC was $10 \%$, which is comparable with study done by Sang et al (10.9\%) and lower than Ansari et al $(16.6 \%)$. The present study shows that the strains were resistant to Ampicillin $94.44 \%$ which was higher than study done by Sang et al $(86 \%)$.

\section{Conclusion}

Bacterial enteropathogen play an important etiological role in acute diarrhea among children aged less than 5 years. The high prevalence of bacterial isolates in this study attributable to those aged below 2 years. Increased risk behaviour for diarrhea especially the indiscriminate putting of objects in the mouth and immature state of acquired immunity are some of the susceptible factors associated with this age group. E. coli constituted majority of bacterial isolates. We have used genotyping method (PCR typing on fecal E. coli flora) for the identification of virulent strains in order to obtain insight into diarrheal disease morbidity and epidemiology of diarrheagenic E. coli in an understudied area in Gujarat.The pathogenic strain identification done by genotyping method at NICED KOLKATA using PCR assay. In which we found three strains EAEC, ETEC and 
EPEC, among which EAEC were significantly more common in diarrheal cases, While prevalence of ETEC and EPEC were found to be less. Prevalence of EAEC is common in developing countries and EAEC infects primarily young children. DEC positive isolates seldom dominated the fecal E. coli flora, but for all DEC types, E. coli flora seemed to be correlated to diarrhea.

Thus, a low proportion of DEC in the fecal E. coli flora may not be of significance for development of diarrhea, but may represent occasional carriage of virulence gene(s) by normal flora strains .We saw higher resistance to commonly prescribed antibiotics and this was associated with irrational use of antibiotics for the treatment of diarrhea. The study highlights the need to explore further the impact of antibiotic on childhood diarrhea and to effectively regulate the use of antibiotics in the management of diarrhea while promoting diarrhea prevention initiatives in the community. Also the government and other health agencies monitor and regulate the use/distribution of antibiotics. We recommends multifaceted approach through more health education by electronics and social media regarding appropriate health seeking, awareness and greater intervention at community level by health worker and peoples in diarrhea prevention, control and treatment.

Fundingnone Competing Interests None Declared

\section{Reference}

[1]. Bhatnagar S, Bhandari N, Mouli UC, Bhan MK, Consensus statement of IAP National Task Force. Status Report on management of Acute Diarrhoea. Indian Paediatric 2004 April 17;4: 335-48.

[2]. Catharine P, Ranoa MD. Cost Reduction in the Treatment of Diarrhoeal diseases by Oral therapy. Phil J. Microbial Infect Dis 1983;13(2):98-108.

[3]. Kosek M, Bern C, Guerrant R, The global burden of diarrhoeal disease as estimated from studies published in between 1992 to 2000. Bull World Health Organ 2003;81:197-204.

[4]. Parashar UD, Hummelman EG, Bresse JS, Miller MA, Glass RI, Global illness and deaths caused by rotavirus Disease in children. EmergInf Dis 2003;9:565-72.

[5]. Morris SS, Black RE, Tomaskovic L. predicting the distribution of under five deaths by cause in countries without adequate vital registration system. Int J Epidemiol 2003;32:1041-51.

[6]. The State of Asia-Pacific`s children 2008( Source : Asian development bank, Inequality in Asia: Key Indicators 2007, Special Chapter Highlights, ADB, Manila,2007 p.8)

[7]. Estimation of burden of diarrhoeal diseases in India. National Institute of Cholera and Enteric Disease Kolkata. (Source: Census of India 2001)

[8]. WHO (1999), health situation in South East Asia Region1994-1997, Regional Office of SEAR, New Delhi

[9]. Willie Kipkemboi Sang, Valerie Oundo, David Schnabel. Prevalence and antibiotic resistance of bacterial pathogens isolated from childhood diarrhea in four proviences of Kenya. J Infect DevCtries 201; 6(7):572-578.

[10]. Ansari S, Sherchand JB, Parajuli K, Mishra SK, Dahal RK, Shrestha S, Tandukar S, PokhretBM.Bacterial etiology of acute diarrhea in children under five years of age.. J Nepal Health Res Counc 2012 Sep; 10(22):218-23.

Tables

Table- 1 Bacteria Isolated Of Different Species

\begin{tabular}{|c|c|c|}
\hline Organism & No. & Percentage \\
\hline E.coli & 108 & $54 \%$ \\
\hline Shigellasonnei & 1 & $0.5 \%$ \\
\hline No Bacterial pathogen & 91 & $45.5 \%$ \\
\hline
\end{tabular}

Table-2 Genotype Pattern Of E.Coli Isolates

\begin{tabular}{|c|c|c|}
\hline Organism & No. & Percentage \\
\hline Eaec & 8 & $80 \%$ \\
\hline Etec & 1 & $10 \%$ \\
\hline Epec & 1 & $10 \%$ \\
\hline
\end{tabular}

Table- 3 Resistance Pattern Of E.Coli Isolates

\begin{tabular}{|c|c|}
\hline Drug & Resistance (\%) \\
\hline Ceftazidime-Clavulanic acid(CAC)30/10ug/disc & $19.44 \%$ \\
\hline Ceftazidime(CAZ) 30ug/disc & $87.96 \%$ \\
\hline Levofloxacin(LE)5ug/disc & $62.03 \%$ \\
\hline Meropenem(MRP)10ug/disc & $1.85 \%$ \\
\hline Cephazolin(CZ)30ug/disc & $91.66 \%$ \\
\hline Amikacin(AK)30ug/disc & $34.25 \%$ \\
\hline Piperacillin-Tazobactam(PTZ)100/10 ug/disc & $19.44 \%$ \\
\hline Cefuroxime(CXM)30ug/disc & $86.11 \%$ \\
\hline Cefepime(CEP)30ug/disc & $29.62 \%$ \\
\hline Cefotaxime(CTX)30ug/disc & $93.51 \%$ \\
\hline Tetracycline(TE)30ug/disc & $86.11 \%$ \\
\hline Cotrimoxazol(COT)1.25/23.75ug/disc & $80.55 \%$ \\
\hline Ampicillin(AMP)10ug/disc & $94.44 \%$ \\
\hline Chloramphenicol(C)30ug/disc & $50.92 \%$ \\
\hline Cephalothin(CEP)30ug/disc & $93.51 \%$ \\
\hline
\end{tabular}

